\title{
Dento-Maxillary Disharmony by Default in Mixed and Permanent Dentition at Odontostomatology Department of Donka National Hospital, Guinea
}

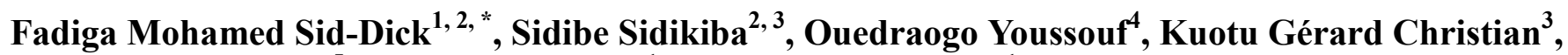 \\ Diouf Joseph Samba ${ }^{5}$, Lama Barthélémy ${ }^{1}$, Djibo Sambou Abass ${ }^{1}$ \\ ${ }^{1}$ Department of Odontostomatology and Maxillo-Facial Surgery, Donka National Hospital, Conakry, Guinea \\ ${ }^{2}$ Faculty of Health Sciences and Techniques, Gamal Abdel Nasser University of Conakry, Conakry, Guinea \\ ${ }^{3}$ Maferinyah National Centre for Training and Research in Rural Health, Forécariah, Guinea \\ ${ }^{4}$ University Hospital Center of Yalgado Ouedraogo, Ouagadougou, Burkina Faso \\ ${ }^{5}$ Faculty of Medicine, Pharmacy and Odontology, University Cheikh Anta DIOP, Dakar, Senegal \\ Email address: \\ fadigamsiddick@gmail.com (F. M. Sid-Dick) \\ ${ }^{*}$ Corresponding author
}

\section{To cite this article:}

Fadiga Mohamed Sid-Dick, Sidibe Sidikiba, Ouedraogo Youssouf, Kuotu Gérard Christian, Diouf Joseph Samba, Lama Barthélémy, Djibo Sambou Abass. Dento-Maxillary Disharmony by Default in Mixed and Permanent Dentition at Odontostomatology Department of Donka National Hospital, Guinea. International Journal of Dental Medicine. Vol. 5, No. 1, 2019, pp. 9-13. doi: 10.11648/j.ijdm.20190501.12

Received: November 17, 2018; Accepted: December 17, 2018; Published: March 14, 2019

\begin{abstract}
Background: Dento-maxillary disharmony is a disproportion between the size or the mesiodistal diameter of the teeth and the perimeter of the corresponding alveolar arches. The objective of this study is to describe frequency and management of default dento-maxillary disharmonies in the department of Odontostomatology of Donka National Hospital. This was a descriptive prospective study with all patients seeking dental care at the department of Odontostomatology of Donka National Hospital. The study was conducted over 13 months from June 2016 to 30 June 2017. Among the 485 patients seeking dental care, the frequency of dento-maxillary disharmonies was $7.21 \%$. The mean age of patients was 8.75 years, with extreme ages of 7 and 26 years. Females were the most represented with $77.15 \%$ that is a sex ratio of 0.29 . The upper arch was the most concerned by the dento-maxillary disharmony with a proportion of $57.14 \%$. The orthodontic treatment was the commonest treatment with $57.14 \%$. Dento-maxillary disharmony had many clinical signs that allowed the practitioner making early diagnosis. Early management could help remodeling the bone genome and reducing the risk of relapse, and the length of orthodontic treatment.
\end{abstract}

Keywords: Dento-Maxillary Disharmony Default, Odontostomatology, Mixed and Permanent Dentition, Conakry

\section{Introduction}

Dento-maxillary disharmony is a disproportion between the size or the mesiodistal diameter of the teeth and the perimeter of the corresponding alveolar arches. It's the commonest cause of orthodontic consultations [1]. Thus, the dento-maxillary disharmony corresponds to the discrepancy between the space necessary for the correct alignment of the set of permanent dentition and the space available on the arch, meaning that on the bone support alveolar. This can be described either by dental default or by dental excess. It can also be isolated or associated with the Angle class malocclusions, which is one of the complications [2].

Very often, an individual can inherit big teeth from one of his parents and small jaws from the other, and vice versa. What introduces the notion of dento-maxillary disharmony (DMD). This disharmony can occur at various stages of the dentition. Whatever the stage of the dentition, the dentomaxillary harmony which allows a balanced occlusion, is one of the way of durability of the mandibular system. Indeed, most of existing oral health measures in dentistry do not apply to orthodontic patients, since the majority of 
orthodontic abnormalities are asymptomatic and associated with aesthetic problems rather than functional problems. However, aesthetic and orthodontic treatment must have an impact on the patient's well-being and quality of life [3]. According to Câmara et al. incisors exposure during speech, at smiling and when the lips are at rest is an important factor in facial aesthetics, as it influences perception of the human face [4]. Moreover, younger individuals have greater maxillary incisor exposure, while older people have greater mandibular incisor exposure. This highlights the aesthetic needs of patients according to age [4]. Thus, when treatment goals are reached in teenagers, the aesthetics and harmony of the smile are generally very satisfying. In addition, regardless muscular dysfunction origin, it must to be noted at the beginning of the orthodontic treatment in order to give an aesthetic prognosis. [5]

In addition, early diagnosis and successful treatment of malocclusions can have both short-term and long-term benefits by achieving the goals of occlusal harmony and function, as well as dento-facial aesthetics [6-9]. Thus, dentists have the responsibility to recognize, diagnose and manage or refer abnormalities of the dentition, depending on the complexity of the problem and the training, knowledge and experience of the clinician [10].

In Guinea, a study on interceptive treatment of alveolar protrusion at Donka National Hospital in 2011 reported that among abnormalities associated with alveolar protrusion, dento-maxillary disharmonies accounted for 46.15\% [11]. Thus, the frequency of observed cases of DMD during consultations and the lack of previous studies on the topic in Guinea were the main motivations to conduct this study.

The aim of this study was to determine the frequency and describe the management of default dento-maxillary disharmonies at the department of Odontostomatology of Donka National Hospital, Conakry, Guinea.

\section{Materials and Methods}

\subsection{Study Design and Population}

This was a 13-month prospective descriptive study from 01 June 2016 to 30 June 2017 conducted in the department of Odontostomatology at Donka National Hospital. The study population consisted of all patients with DMD problems diagnosed in the department during the study period.

\subsection{Data Collection and Study Variables}

An exhaustive census of patients with default dentomaxillary disharmonies was performed among all patients seeking dental care in the department Odonto Sstomatology during the study. Thus, we recorded 485 patients with indication of orthodontic abnormalities among which 35 cases of DMD. Data were collected from patients' medical records, using a structured questionnaire.

Study variables included the demographic characteristics (age, sex), reasons for consultation, clinical characteristics (type of dentition, arch concerned, location of abnormality by sector, and associated abnormalities) and treatment (type of treatment performed).

\subsection{Data Analysis}

Statistical analysis was performed using SPSS Statistics 20.0. Descriptive variables were presented as proportions or mean (with standard deviation) with a 95\% confidence interval.

\subsection{Ethical Considerations}

The protocol of this study was approved by the Scientific Committee of the Department of Odontology, Faculty of Health Sciences and Techniques, University of Conakry. Informed consent was obtained from each patient or patient's parent prior to data collection.

\section{Results}

During the study, 485 received patients with indications of orthodontic abnormalities among them, 35 patient traited for dento-maxillary disharmony that is $7.21 \%$ compared to $92.79 \%$ for other pathologies.

\subsection{Socio-Demographic Characteristics of the Sample}

Among the 35 patients with default dento-maxillary disharmonies, girls with 27 cases $(77.2 \%)$ were more represented compared to boys ( 8 cases or $22.9 \%$ ) with a sex ratio of 0.29 . The average age of the patients was $8.8 \pm 7.4$ with ages ranging from 7 years to 26 years. The most represented age group was $7-11$ years $(60.0 \%)$ followed by the age group of $12-16$ years $(17.1 \%)$ and $22-26$ years (14.2\%). Patients aged 17 to 21 years were the least represented $(8.6 \%)$.

\subsection{Breakdown According to the Reasons for Consultation and Types of Toothing}

Of the 35 patients included in the study, 28 people $(80 \%)$ consulted for aesthetic problems, whereas 7 patients $(20 \%)$ consulted for functional and aesthetic problems.

As for the types of dentition found after examination, $60 \%$ ( $n=21$ patients) of patients had mixed dentition versus $40 \%$ ( $\mathrm{n}=14$ patients) with permanent dentition.

The maxillary arch was the most affected by DMD (-) with a proportion of $62.86 \%$ followed by the mandibular arch (37.14\%). The previous sector with $54.28 \%$ was the most concerned by the DMD (-) followed by the associated front and side sectors $(20 \%)$ and the lateral sector (11.42\%) (Table 1).

Table 1. Distribution of Patients by Location of DMD (-) and Arch Areas of patient with dento-maxillary disharmony at the department of Odontostomatology of Donka National Hospital, Conakry (Guinea). June 2016 to 30 June $2017(n=35)$.

\begin{tabular}{lll}
\hline & Number & Percentage \\
\hline Arcades $(\mathrm{n}=35)$ & & \\
Maxillary & 22 & 62.86 \\
Mandible & 13 & 37.14 \\
Sectors $(\mathrm{n}=35)$ & & \\
\hline
\end{tabular}




\begin{tabular}{lll}
\hline & Number & Percentage \\
\hline Anterior & 19 & 54.28 \\
Anterior + Lateral & 7 & 20.02 \\
Posterior & 5 & 14.28 \\
Lateral & 4 & 11.42 \\
\hline
\end{tabular}

Of the 35 patients with MDD (-), 20 patients with associated abnormalities and alveolar protrusion were the most dominant (55\%). (Table 2)

Table 2. Distribution according to the associated anomalies of patient with. dento-maxillary disharmony at the department of Odontostomatology of Donka National Hospital, Conakry (Guinea). June 2016 to 30 June 2017 $(n=35)$.

\begin{tabular}{lll}
\hline Associated anomalies & Number $\mathbf{N}=\mathbf{2 0}$ & Percentage \\
\hline Alveolar protrusion & 11 & 55.00 \\
Class II.1 & 4 & 20.00 \\
Class II.2 & 2 & 10.00 \\
Exo-alveoli & 2 & 10.00 \\
Class III & 1 & 5.00 \\
\hline
\end{tabular}

Depending on the type of treatment, $57.14 \%$ of patients received orthodontic treatment versus $42.86 \%$ of orthodontic treatment (Table 3). Treatment duration ranged from 12 to 24 months in the patients in the sample.

Table 3. Distribution by type of treatment of patient with dento-maxillary disharmony at the odontostomatology department of Donka National Hospital, Conakry (Guinea). June 2016 to 30 June 2017 (n=35).

\begin{tabular}{lll}
\hline Treatment & Number & Percentage \\
\hline Orthodontic & 20 & 57.14 \\
Surgical-orthodontic & 15 & 42.86 \\
\hline
\end{tabular}
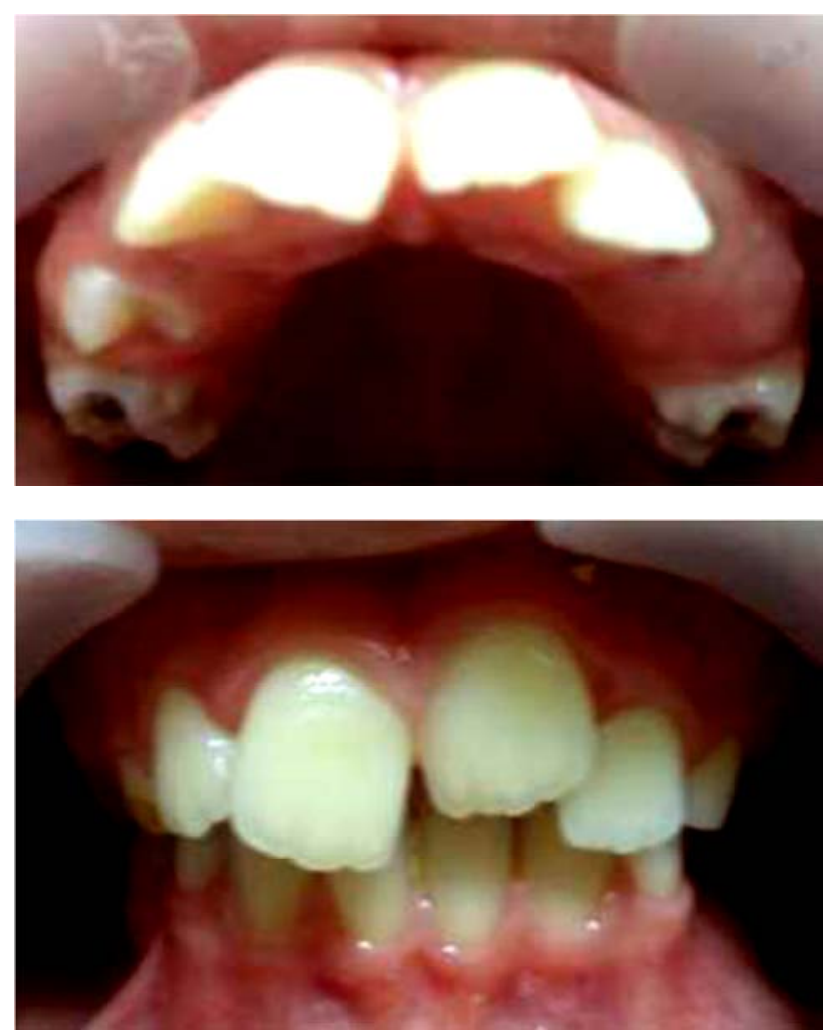

Figure 1. 11-year-old patient presenting maxillary dento-maxillary disharmony by default of the anterior sector. Before treatment.
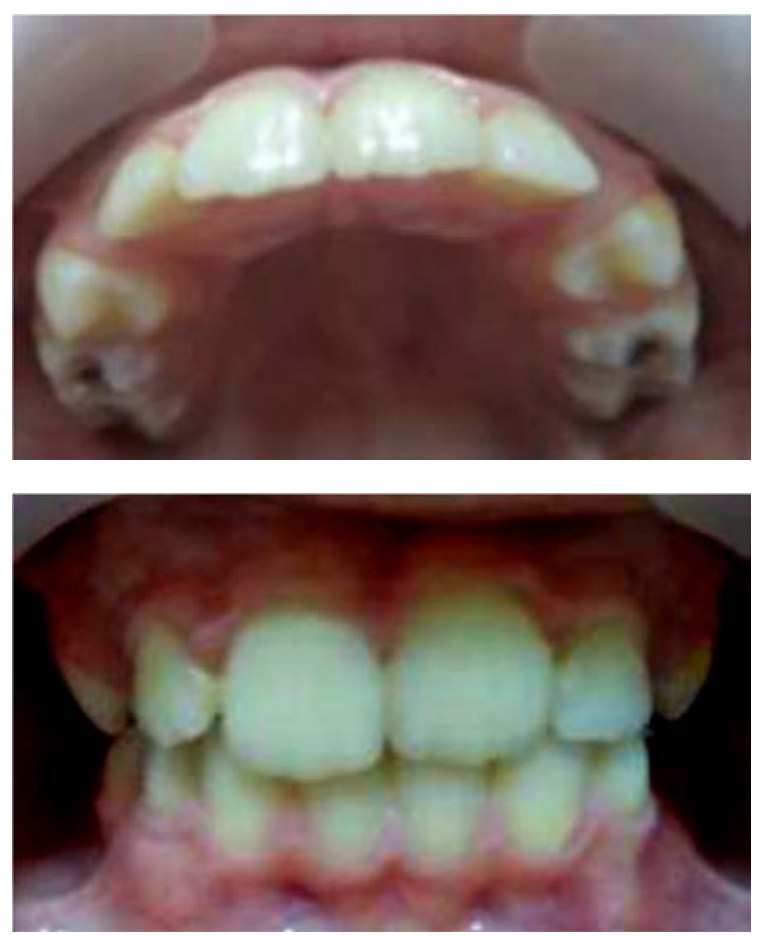

Figure 2. Orthodontic appliance in the mouth 2 months after the start of treatment.

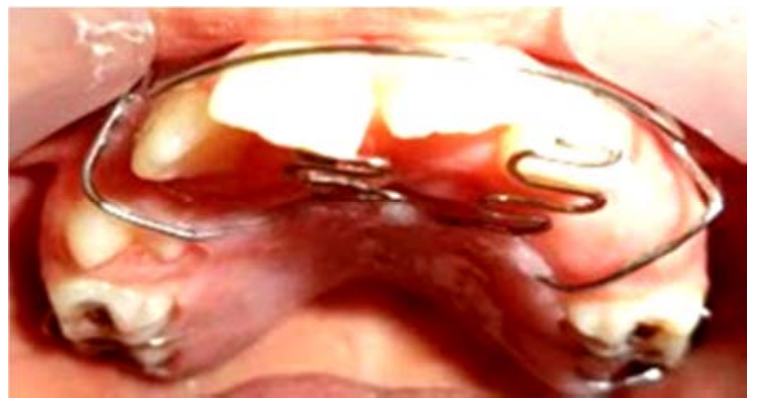

Figure 3. End of treatment 12 months later.

\section{Discussion}

This study is the first of its kind to describe the frequency and management of dento-maxillary disharmonies by default at the department of Odontostomatology of Donka National Hospital in Guinea. The frequency of dento-maxillary disharmonies in our sample was relatively low (7.21\%) among patients seeking health care at the Odonto Stomatology Department of Donka National Hospital in Conakry. This observed low frequency could be explained by the fact that the population is often not informed of the possibilities of treatment of anomalies, hence the need to inform and sensitize the population on the possibilities of care. Higher frequency of dental disharmony was reported in Iran, in the Orthopedic Dento-Facial department of the faculty of Shiraz, where DANAIE et al. in 2006 found the prevalence of $47.9 \%$ among 3776 children aged from 7 to 9 years old [15]. Similar findings showing higher proportion of dento-maxillary disharmonies among patients were reported by other studies conducted in Nigeria by ONYEASO (2002) 
[13], ONYEASO et al. (2004 [14] and OLURANTI et IFEOMA, (2009) [15]. A study of 636 Nigerian children aged from 12 to 17 years old showed that about $20 \%$ had dental crowding [13]. ONYEASO et al. evaluated 289 orthodontic patients who had visited Dental Centre, University College Hospital in 2004. They reported that percentage of dental crowding was $29.7 \%$ among patients [14].

Among patients with dento-maxillary disharmony, females were the most represented $(77.15 \%)$ with a sex ratio of 0.29 . This could be explained by the fact that, girls are more funof aesthetic than boys. The finding reported in our study contrasts with the one found by Yassir Talal OMER et al [3] in a Lebanese population in 2016, whith a male predominance $(54 \%)$.

The most represented age group in the sample was 7-11 $(60 \%)$. The predominance of this age group is explained by the fact that the majority of patients were in mixed dentition, which would be favorable for orthopedic or orthodontic treatment.

The findings of this study also showed that the most dominant reason for consultation in patients was aesthetics (80\%). This result is higher than the one found by Clément $\mathrm{A}$ et al, who, in their study of the psychic dimension in orthodontic procedures, reported a proportion of $74 \%$ of consultation reason for aesthetics [16]. The high proportion of consultation for the aesthetics could be justified by the fact that most of the patients seeking health care visited at the Odonto Stomatology Department of Donka for aesthetic reasons.

The present study found that the mixed dentition was the commonest $(60 \%)$ type of dentition among study participants. This result could be explained by the fact that during the periode of 7 to 11 years, transient abnormalities are frequent because of the cohabitation of permanent teeth (which have important mesio-distal diameters) with temporary teeth.

Regarding localization, the maxillary arch was the most affected by the dento-maxillary disharmony (57.14\%) compared to the mandibular arch (37.14\%). OLURANTI. O. $\mathrm{D}$ and IFEOMA in Nigeria reported contrary results: $38.7 \%$ maxillary congestion versus $43.2 \%$ mandibular crowding among 633 patients seeking dental care [15].

Of the associated abnormalities, proalveolysis was the commonest (55.0\%) followed by Class II.1 (20\%), Class II.2 $(10.0 \%)$ and Class III $(5.0 \%)$ abnormalities. This result is contrary to that reported by Matysiak. M et al [17] in 2003, who found abnormalities associated with DMD (-) in the following proportions: $59.9 \%$ for class II.1, 23.4\%, for class II. 2, 22, 3\% for class III and $19.8 \%$ for alveolar anomalies.

Orthodontic treatment was the most used for MDM management (57.14\%). Anne Marie Rauten et al [18] reported similar result with $74.87 \%$ orthodontic treatment among the study participants. These results could be justified by the fact that for most patients the indication of orthodontic treatment is much more important than that of orthodontic treatment. The duration of treatment for study patients ranged 12 to 24 months. This result is different from that found by Matysiak. M et al who reported that dentofacial orthopedic performed treatments in four or five semesters while taking into account discontinuations [17].

The main limitation of our study is that it was a hospital study, limited to one hospital. It is therefore difficult to estimate the frequency of dental disharmony in the general population. However, this study was conducted in a reference hospital in the country and our results provide basic information that can guide future research and interventions to improve the management of dental disharmony.

\section{Conclusion}

Dento-maxillary disharmony presents many clinical signs that allow early diagnosis. The type of disharmony, its severity and its etiology guide the therapeutic choice between the management of the space, the expansion of the arches or the realization of planned extractions. Early management could interfere with bone remodeling that can change the expressiveness of the bone genome, reduce the risk of relapse, and reduce the length of orthodontic treatment.

\section{Authors' Contributions}

FMS, SS and OY were involved with conception and design of the protocol. FMS, SS, and NPI were involved with acquisition of data. SS and KGC did the data analysis and all authors were involved with interpretation. All authors have given approval for the final version to be published and are accountable. All authors read and approved the final version.

\section{Conflict of Interest}

The authors declare that they have no competing interests and no support was received from any funding agencies, public or private.

\section{References}

[1] Regragui S, Dakka A, Regragui F, Bouklouz A, Zaoui F. Calculation of dentomaxillary dysharmony (DMD): which measurement method? Int Orthod. 2010; 8 (1):64-77.

[2] Laure Achour-Sabbah. Early signs of dento-maxillary dysharmony. Life Sciences [q-bio]. 2017. <dumas-01477853.

[3] Omer YT, Bouserhal J, Hawas N, Abdel Moneim El Sayed A. Association between normative need for orthodontic treatment and self-perceived need in a population. libanaise. Int Orthod. 2016; 14 (3):386-98.

[4] Câmara CA, Martins RP. Functional Aesthetic Occlusal Plane (FAOP). Dental Press J Orthod. 2016 July-Aug; 21 (4):114-25. DOI: http://dx.doi.org/10.1590/2177-6709.21.4.114-125.sar. Dental Press J Orthod. 2016 July-Aug; 21 (4):114-25.

[5] Muller Christine, Alouini Ons, Chouvin Monique. Objective smile: and if one interested in the lips?. Orthod Fr 2018; 89:21-40. EDP Sciences, SFODF, 2018. https://doi.org/10.1051/orthodfr/2018003. 
[6] Kanellis MJ. Orthodontic treatment in the primary dentition. In: Bishara SE, ed. Textbook of Orthodontics. Philadelphia, Pa: WB Saunders Co; 2001:248-56.

[7] Woodside DG. The significance of late developmental crowding to early treatment planning for incisor crowding. Am J Orthod Dentofacial Orthop 2000; 117 (5): 559-61.

[8] Kurol J. Early treatment of tooth-eruption disturbances. Am J Orthod Dentofacial Orthop 2002; 121 (6):588-91.

[9] Sankey WL, Buschang PH, English J, Owen AH III. Early treatment of vertical skeletal dysplasia: The hyperdivergent phenotype. Am J Orthod Dentofacial Orthop 2000; 118 (3):317-27.

[10] American Academy of Pediatric Dentistry. Policy on ethical responsibility to treat or refer. Pediatr Dent 2013; 35 (special issue): 106 .

[11] Fadiga MS, Traore N, Camara SAT, Lama B, Beavogui D. Orthodontic anomalies from anteroposterior sense in JOZIP BROZ TITO primary school of Belle Vue. Medical Guinea. 2008; (62):41-7.

[12] Danai et al: Distribution of malocclusion types in 7-9 year old Iranien children. East Mediterr health. 12 (1-2); 236-40; 2006.

[13] Onyeaso CO. Prevalence of malocclusion among adolescents in Ibadan, Nigeria. Am J Orthod Dentofacial Orthop. 2004 Nov; 126 (5):604-7.

[14] Onyeaso CO, Aderinokun GA, Arowojolu MO. The pattern of malocclusion among orthodontic patients seen in Dental Centre, University College Hospital, Ibadan, Nigeria. Afr J Med Med Sci. 2002 Sep; 31 (3):207-11.

[15] Oluranti OD, Ifeoma LU. Referral mode and pattern of malocclusion among patients attending the Lagos University Teaching Hospital, Lagos, Nigeria. Odontostomatol Trop. 2009 Dec; 32 (128):17-23

[16] Clément A, Guilyardi H, Makaremi M, Goudot P, Schouman $\mathrm{T}$. The psychic dimension in surgical-orthodontic protocols. Rev Orthopédie Dento-Faciale. 2016; 50 (1):7-20.

[17] Matysiak M, Brochard P, Fouilleul T, Merentier J, Peyrot B, Rouch M. Orthopédie dento-faciale : frequency, geographical distribution and duration of treatment of dento-maxillary dysmorphosis. National study using data from the general sickness insurance plan. Rev Medical Assur Mal. 2003; 34 (1):23-32.

[18] Rauten A-M, Şurlin P, Olteanu M, Maglaviceanu C, Popescu MR, Teodorescu E. Clinical and therapeutic aspects in dentoalveolar disharmony (DAD) with crowding. Curr Health Sci J. 2013; 39 (1):39-44. 